Voix et Images

voixetimages

\title{
Les Signes de Rina Lasnier
}

\section{Sylvie Sicotte}

Volume 4, numéro 1, septembre 1978

Rina Lasnier

URI : https://id.erudit.org/iderudit/200134ar

DOI : https://doi.org/10.7202/200134ar

Aller au sommaire du numéro

Éditeur(s)

Les Presses de l'Université du Québec

ISSN

0318-9201 (imprimé)

1705-933X (numérique)

Découvrir la revue

Citer cet article

Sicotte, S. (1978). Les Signes de Rina Lasnier. Voix et Images, 4(1), 33-38.

https://doi.org/10.7202/200134ar

Ce document est protégé par la loi sur le droit d'auteur. L'utilisation des services d'Érudit (y compris la reproduction) est assujettie à sa politique d'utilisation que vous pouvez consulter en ligne.

https://apropos.erudit.org/fr/usagers/politique-dutilisation/
Cet article est diffusé et préservé par Érudit.

Érudit est un consortium interuniversitaire sans but lucratif composé de l’Université de Montréal, l'Université Laval et l'Université du Québec à Montréal. Il a pour mission la promotion et la valorisation de la recherche. https://www.erudit.org/fr/ 


\section{Les Signes de Rina Lasnier}

Étoiles de mots, îlots d'émotion, baisers d'accueil et d'adieu, les poèmes de Rina Lasnier jaillissent d'une étreinte charnelle avec la vie. Ils mordent dans la parole avec la jouissance douce-acide éprouvée par celui qui mord dans la pomme. Ils réussissent à dire l'indicible.

Rapprochement à prime abord inattendu, j'ai eu le sentiment, en écoutant le dernier disque de Jacques Brel, de lire du Rina Lasnier. II atteint à ce qu'elle exprime depuis longtemps : cette fulgurance du verbe, cette passion pour la vie au cœur de la mort, cette puissance de l'amour chez les amants qui se retiennent par les yeux. Mettant l'accent sur le tragique de la vie, il ne parvient pas cependant à cette perception de l'invisible qui éclaire les Signes ${ }^{1}$ et qui résulte de la conscience du mouvement au cœur de l'immobilité.

Dans les Signes, l'être humain devient œil cosmique. II regarde l'univers et l'œil de l'univers lui rend son regard. "L'invisible nous regarde. [...] L'Esprit passe l'anneau agrandi du firmament, resserre son erre, et dans l'eau ronde d'un regard, franchit son infranchissable... ${ }^{2}$,

Regardant regardé, l'homme, dans le poème liminaire intitulé aussi "les Signes", se perçoit "geste inachevé à profil de beauté" et il instaure une relation amoureuse avec la terre, relation qui a la mort comme nœud intime.

Ce qui m'émeut dans ce livre, c'est qu'il témoigne d'un apprivoisement de la mort sentie, non pas comme un scandale, mais comme un moyen d'atteindre "la flamme des choses".

Toute matière au creuset de son chiffre fait place à la flamme des choses ${ }^{3}$;

La mort, dans "les Signes", est "éclipse du temps", macération au sein de la terre dans «la Race des pluies vertes», dénouement en une constellation de «signes". Évocation des feux-follets de la légende, ces 
signes qui ont tout d'abord une connotation visuelle, deviennent expression de l'éternel par leur conjonction avec le rythme:

Toute musique qui macère ta poussière

fulgure en toi la note nomade de l'éternel;

L'éclipse du temps, associée à l'hiver et à la neige, est provoquée par notre propre cécité devant la lumière solaire, par notre propre cécité devant la fuite des jours. Et cet aveuglement de l'homme se répercute dans la "Lassitude du soleil »:

Mancuvrier de la lumière incorruptible,

ta course lasse est de sortir du jour,

de passer outre à l'œuvre fuyante des formes,

d'arracher ton âme avant qu'advienne l'Ange... ${ }^{4}$

La poésie de Rina Lasnier tente de fixer une fuite, de réconcilier le temps qui est rond, cyclique, avec le temps qui est linéaire et qui a pour but un absolu irréversible. Elle illustre graphiquement la conjoncture de deux astres. Dans cette première section du livre intitulée "le Règne", le soleil reproduit la linéarité, la terre, elle, est cercles concentriques.

Soleil né de la fronde d'une lumière verbale ${ }^{5}$,

Terre, vieille bible récrite de main multiforme, parole variable dans l'anagramme des vents; image du temps passageant les éternités ${ }^{6}$.

Le terme passager lui-même évoque la circularité du manège et transforme en recommencement ce qui était cheminement vers une fin. II appelle par juxtaposition la figure de l'anneau qui servait de véhicule à l'Esprit dans "L'invisible nous regarde». Mais l'anneau de ce poème-ci, "le Règne", n'est plus seulement associé au regard, mais à la chair.

Mais l'anneau incrusté dans la chair-glaise

ajuste le chiffre divin au germe continuel.

C'est au sein de la chair que vibre la contradiction, droite-cercle. Mais la chair elle-même est intimement liée à la glaise, l'éternel humain a une densité de cendre. L'être humain rejoint l'infini en épousant intimement la terre. Même si les galaxies forment le cercle ultime de notre sensibilité, "une seule mort animale fissure plus la nuit" que "la passion des astres ${ }^{7}$ ". II n'est de recours, en définitive, que vers "Notre terre".

Notre terre nous retient par l'abondance de ses formes.

Règne de la terre par le lait, le sang et le vin, royaume ouvert par l'insecte et la foudre, refermé par la clef de voûte de l'oiseau; terre nôtre, et le Père n'est plus sauvage ${ }^{8}$.

En même temps que déchiffrement des signes, ce livre est un chant d'amour vers la terre. Dans les premiers recueils, la femme toute arrondie de chair, essayait de dépouiller l'arbre terrestre de ses feuilles, dans 
l'espoir d'apercevoir un peu de ciel, de découvrir la structure cachée sous toute cette luxuriance. Dans ce dernier livre, la femme réduite à ses os, retrouve toute cette chair qui l'habitait, par l'intermédiaire de la terre. Elle apprivoise cette terre qui sera sa chair.

la terre s'assied à la paille de ses collines

et rêve la fleur carnée et le fruit fluide?

La terre refait le cycle de la vie, de la fleur au fruit. Le poète lui, rêve à la chair de la fleur et à la fluidité du fruit, chaque cycle entremêlant ses qualités, la fleur ayant déjà la chair du fruit, le fruit étant déjà énergie dispersée dans un autre organisme.

Et ces villes "carapaces" et ces "mers ovales" et ces "eaux batailleuses" et ces "saisons migratrices" font la vitalité d'une terre “ancrée de pierres" et "livrée aux vignes vierges des racines ${ }^{10}$ ".

Une évolution a eu lieu entre ce chant d'amour à notre terre et ces poèmes du début où la terre était associée au mal et les racines au serpent tentateur. Ici la terre est vie. Tout d'abord servante taciturne associée aux ombres puis jeune épousée promise aux métamorphoses et enfin «terre forestière arquée de bras tendus bas" la terre a un médiateur qui est "l'arbre et l'homme". Elle prend figure de femme avec sa "paupière de neige ", "plus pure que la soie relevée du bouleau blanc ${ }^{11}$ ".

Rina Lasnier, tout au long de son œuvre, a constamment parlé de l'amour, mais a rarement écrit dans un poème: je t'aime. Ici elle le dit, mais c'est à la «Terre noire " qu'elle s'adresse.

Je t'aime du cœur noir de ta terre franche de la force des blessures fusionnées;

la nuit naît du sang corporel de la terre, mon visage, de sa pure perte entre tes mains;

il y a moins de nuit dans le haut des astres que de douleur dans les ruisseaux de la mémoire;

soude-moi sans peur aux terres fortes d'aimer:

l'eau noire de mes yeux n'a pas de larmes

mais ce face à face de la pierre et de ta nuit... ${ }^{12}$

Elle affirme l'identité de l'amour et de la nuit à travers ce cceur noir de la terre, elle affirme la force qui a traversé la blessure, la division. Le visage de chair se reformera de sa décomposition organique, la nuit véritable surgira du corps humain de la terre. L'amour renaîtra de la perte du moi personnel.

Par la force de la fusion avec le ccour noir de la terre, l'eau des yeux devient noire. II s'agit de percer cette alchimie du noir, de faire ressortir cette puissance du noir qui est absorption complète de la lumière, qui est trop-plein de lumière. Seule une force sans défaillance pénétrera ce noir de pierre, ce noir de nuit, ce noir de terre. 
La douleur est comprise dans cet amour noir. "Sait-on la douleur de la matière en fusion passant à la dure solitude de l'inertie et du froid ${ }^{13}$ ?" Mais la douleur est force d'amour, elle surmonte la peur faiblarde. Le mot souder traduit fermement cette volonté ultime de l'amour total dans la nuit totale.

L'écriture poétique est aussi, expression d'amour à travers la nuit, par son pouvoir de divination. "Il arrive souvent que la poésie, aveugle scrutant la nuit, se fasse malgré elle, prophétique. Ainsi, la douleur d'un autre, partagée dans le poème, devient solidairement et solitairement nôtre, juste un peu plus tard dans la nuit ${ }^{14}$."

Ce livre qui s'est ouvert sur le givre et sur l'homme dénoué, " constellé de signes", se referme sur le vin et sur la "Nuit de l'amante", cette tension vers l'amour parfait, tension inhérente à toute solitude, réaffirmant cette aspiration profonde de l'être vers la nuit parfaite.

le vase renaît du vin, la bague de l'aubier, la nuit parfaite, d'une ombre épousable... ${ }^{15}$

Rina Lasnier, dans les Signes, utilise la prose pour commenter une quarantaine de vers choisis dans ses livres précédents. Elle veut expliciter la difficulté pour le poète de faire le choix entre le bon grain et l'ivraie. Car l'ivraie abandonnée n'est pas que déchets. Elle est inextricablement mêlée aux germes d'une moisson nouvelle.

Le poète «sait, parfois avec angoisse, que chaque naissance du poème provoque spontanément l'avortement de combien de poèmes parallèles et différents? En effet, le moi lyrique est à la fois polyphonique et fuyant comme la mer, vague sur vague, de surface en profondeur. Qu'est-ce qu'un poème sinon la stimulation momentanée de l'esprit, une sorte de houle abordant ses limites par le gonflement de l'inexprimable. Et toute la mer stable semble a jamais perdue avec son plancton de mots ${ }^{16}$ ".

Dans cette section intitulée "Poésie nombreuse", où prose et poésie sont mêlées, l'auteur redit la difficulté de maîtriser le métier poétique, "la dureté de l'apprentissage toujours recommencé», "le qui-vive de la beauté 17 ".

II décrit ce moment de joie libre où se fixe le poème à l'épicentre, "(l'épicentre, c'est l'âme survoltée) ${ }^{18}$ ", ce moment de regret aussi, où beaucoup de richesses restent insaisies. Mais l'acte de création a dilaté l'être du poète è c'est «cette dilatation de l'être qui le renverra à la chasse du poème avorté, recouché dans la mémoire fastueuse de la poésie ${ }^{19}$ ".

"ll cherchera une nouvelle aimantation, une naissance que certes il ne peut volontairement produire, mais qu'il suscitera par cette extrême attention à ce murmure musical d'un amour ancien et toujours répondant ${ }^{20}$." Rina Lasnier termine son avant-dire en réaffirmant le pouvoir 
de l'ombre. "La parole poétique est toujours vivipare, et plus elle est mystérieusement ombreuse, plus elle est nombreuse ${ }^{21}$."

Elle commente plus loin cette nécessité de l'ombre dans une note ajoutée à un vers de la Salle des rêves, "l'éternel se dissout dans l'intarissable»: «L'unité d'un poème est autant faite d'obscurité que de clarté; ainsi la nuit avec ses fonds ténébreux et ses lumières astrales, pareilles aux rêves du sommeil profond. Peut-être qu'en poésie, dans le désistement de tout gain, l'intarissable et l'éternel s'éveillent-ils l'un à l'autre... ${ }^{22}$ "

«Poésie nombreuse» permet une approche plus facile de la poésie sans fissure de Rina Lasnier. Dans “le Pavillon de la joie", par exemple, elle nous fait assister au moment de vie intense qui a précédé la rédaction d'un poème. Dans une prose qui a déjà une résonance poétique, elle nous entraîne avec elle dans le petit jour et plante le décor qui abritera ce moment de joie parfaite. Le poème qui suit immédiatement, et qui reprend ce même moment, est analogue à ces tabernacles que l'on trouve en Orient, "fermés sur des papillons sacrés parce que marqués du sceau de la beauté parfaite ${ }^{23}$ ". Il brille de tous les feux de la poésie, mais il est aussi plus fermé que la prose précédente quant au sens immédiatement saisissable.

On voit confirmée, dans aPoésie nombreuse", l'importance du thème de l'arbre pour Rina Lasnier. "Le thème récurrent de l'arbre ne m'a jamais paru une réitération, mais une force profonde; peut-être celle d'une forme très familière, et la poésie appelée à cette forme fixe pourrait se diversifier comme le rêve ramifié selon le mythe. La forêt, barreaux volontaires, mais aussi dôme ouvert sur l'imaginaire ${ }^{24}$ ».

En effet, cet arbre “d'or vert", véhicule de l'anneau et du regard, unifie le foisonnement de l'indéterminé; il catalyse les images à l'épicentre. "ll dit sans effort le passage immuable de l'Invisible ${ }^{25}$." Et la poésie de Rina Lasnier se situe dans ce nœud: passage immuable, mouvement immobile.

«L'arbre, flamme rugueuse de l'amour inépuisablement vert, et sa paumée d'ombres songeuses nous rafraîchit de l'active brûlure d'aimer ${ }^{26}$." A l'image de l'arbre, la parole poétique de cet auteur unit flamme et verdure, rugosité et douceur, amour et songe en une source infiniment jaillissante; elle mêle le bref et le long, le jour et la nuit et ce qui semblait s'opposer se transforme en une réalité autre, inédite.

L'arbre qui étreint une “ondée d'oiseaux", "referme la pensée de l'espace ${ }^{27}$ ". Ne pourrait-on pas dire, en dernière analyse, que l'œuvre de Rina Lasnier, qui explore le cœur de la conscience, est silence intarissable? 
1. Les Signes, poèmes, Montréal, Éditions Hurtubise HMH, «Sur parole», 1976.

2. «L'Invisible nous regarde", p. 109.

3. "Les Signes", p. 9.

4. "Lassitude du soleil ", p. 16.

5. Ibid.

6. "Le Règne", p. 13.

7. «Les Galaxies», p. 17.

8. «Notre terre", p. 18.

9. Ibid.

10. Ibid.

11. Ibid.

12. "Terre noire», p. 113.

13. "Note ", p. 98.

14. «Noten, p. 106.

15. "Nuit de l'amante", p. 126.

16. "Poésie nombreuse", p. 78.

17. Ibid.

18. Ibid.

19. Ibid., p. 79 .

20. Ibid.

21. Ibid., p. 80.

22. "L'éternel se dissout dans l'intarissable», p. 108.

23. "Pavillon de la joie $"$, p. 116.

24. "Thème de l'arbre", p. 81.

25. "Signes de l'arbre", p. 84.

26. Ibid., p. 83.

27. "Les Oiseaux de l'orme», p. 103. 